\title{
Brain Tumor Segmentation through Region-based, Supervised and Unsupervised Learning Methods: A Literature Survey
}

\author{
Muhammad Zawish ${ }^{1}$, Asad Ali Siyal ${ }^{2 *}$, Shahzad Hyder Shahani ${ }^{1}$, Aisha Zahid Junejo ${ }^{1}$, Aiman Khalil ${ }^{1}$ \\ ${ }^{1}$ Department of Computer Systems Engineering, Mehran University of Engineering \& Technology (MUET), \\ Jamshoro 76062, Sindh, Pakistan; ${ }^{4}$ Department of Biomedical Engineering, Mehran University of \\ Engineering \& Technology (MUET), Jamshoro 76062, Sindh, Pakistan \\ siyalasadali@gmail.com
}

\begin{abstract}
Image segmentation is one of the most trending fields in the domain of digital image processing. For years, researchers have shown a remarkable progress in the field of Image Segmentation, precisely, for brain tumor extraction from various medical imaging modalities including X-Ray, Computed Tomography and most importantly, Magnetic Resonance Images (MRI). In these medical imaging modalities, accurate and reliable brain tumor segmentation is extremely imperative to perform safe diagnose, healthy treatment planning and consistent treatment outcome evaluation in order to understand and cure the complexities of chronic diseases such as Cancer. This paper presents various image processing techniques that are currently being used for brain tumor extraction from medical images. Though some great work has been done in this domain but none of the techniques has been widely accepted to be brought into practice in real time clinical analysis. The paper concludes with proposing some solutions that would aid in refining the results of the techniques which will lead to clinical acceptance of these computer aided methods.
\end{abstract}

Keywords: Image Analysis, Image Segmentation, Brain Tumor Detection Region Based, Supervised Learning, Unsupervised Learning, Clustering, Watershed Segmentation, Convolutional Neural Networks, SVM, K-Means Clustering, MRI, CT scan

\section{Introduction}

The brain is undeniably considered as the most vital organ in the human body. Although, it only weighs about 3 pounds but works as a central processing unit of human body. It controls and commands the voluntary as well as involuntary actions and reactions in a way that allows us to think and feel, retain memorable moments and feelings. Most importantly, this is the indispensable organ that completes us and turns us into a human [1]. Human brain has been under study since ages due to its complex structure and natural placement inside the skull which hinders the perfect and ultimate diagnosis of the diseases. Naturally, human brain is tightly safeguarded but prone to harms and can be affected by several fatal diseases [1]-[2]. An unwanted mass which is result of irregular growth of tissues (figure 1) - benign and malignant tumors inside the human skull, in some cases, surrounds the brain is known as Brain Tumor. Many different types of brain tumors have been observed and are categorized based on their characteristics. Depending upon their characteristics, brain tumors are categorized into two broad 
Muhammad Zawish, Asad Ali Siyal, Shahzad Hyder Shahani, Aisha Zahid Junejo, Aiman Khalil; Brain Tumor Segmentation through Regionbased, Supervised and Unsupervised Learning Methods: A Literature Survey. Journal of Biomedical Engineering and Medical Imaging, Volume 6, No 2, April (2019), pp 8-26

classifications: noncancerous (benign) or cancerous (malignant) types. Figure 1 shows MRI images of the brain for both of the aforementioned categories. Brain tumors mainly originate inside brain (primary brain tumors), or it can invade the brain by the means of spread of cancer through other parts of the body (metastatic or secondary brain tumors) [2-4].

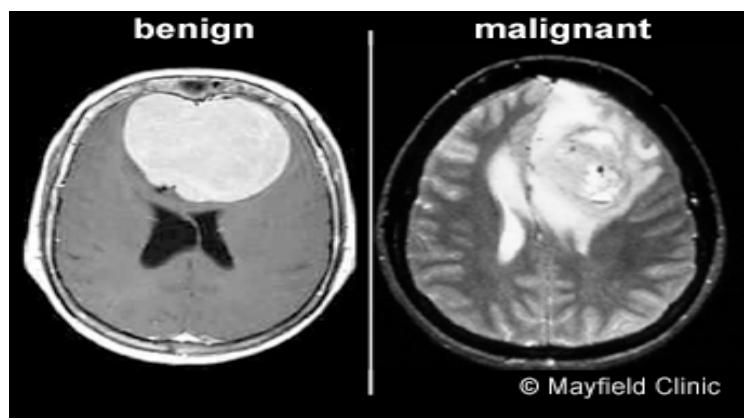

Figure 1: Benign and Malignant Tumors.

However, the growth or spread of brain tumor can be analyzed by the various grades [4] - [5]:

- Grade I:

These are "low grade tumors", which grow at a quite slower rate and exhibit benign morphology at external observation. These tumors look pretty similar as normal cells and have quite lower recurrence of tumor returning or occurrence of spread to other parts of the body. These kinds of tumors are less serious which can be treated with minimal surgery.

- Grade II:

These are also regarded as "low grade tumors", which grow at a quite slower rate, look slightly abnormal and exhibit benign morphology at external observation. However, once removed from the body these tumors can more likely grow again and spread to other parts of the body, if safety precautions are not taken accordingly.

\section{- Grade III:}

These are "high grade tumors", which grow at a quite faster rate and exhibit malignant morphology at external observation. These tumors look like abnormal cells and have quite higher recurrence of tumor returning and spread to other parts of the body frequently. These kinds of tumors are serious which cannot be only treated with minimal surgery, thus require extensive treatment as well such as chemotherapy or radiotherapy etc.

\section{- Grade IV:}

These are also regarded as "high grade tumors", which grow at a quite faster and frequent rate, look abnormal and exhibit malignant morphology at external observation. However, once removed from the body these tumors still have higher probability to grow again and spread to other parts of the body, if safety precautions are not taken accordingly. These kinds of tumors are highly serious which require extensive treatment such as chemotherapy or radiotherapy etc. 
For the treatment of brain tumor in any of the above grades, it must be detected timely and located accurately [4]-[7]. When this anomalous mass occurs inside the brain, it needs to be identified as soon as possible, and for this, various imaging modalities such as Magnetic Imaging Resonance (MRI) and Computed Tomography (CT) are widely used to detect the tumor. Among these medical imaging modalities, MRI is most widely used and highly preferred non-invasive technique in biomedical, radiology and medical imaging fields due to its capability to detect and visualize finer details in the internal structure of the body by generating three dimensional high resolution detailed anatomical images without the use of any damaging radiations. [8]-[10]. The brain MRI segmentation into several brain tissues such as gray matter (GM), white matter (WM) and cerebrospinal fluid (CSF) is highly essential for the diagnosis of various diseases. This technique is basically used to detect the detailed differences in the tissues in noninvasive fashion which have not been examined by other imaging techniques such as Computed Tomography [11].

Once the MRI scanned images are obtained, the goal is to extract out the desired information in order to diagnose the tumor without any delay. One of the major problems in this whole process is separating the abnormal cells from the rest of the image content which is known as the process of segmentation. The manual segmentation is quite challenging as well as time consuming task due to complex structure of the brain and absence of well-defined boundaries among different brain tissues. Therefore, the accurate and reliable brain tumor segmentation is extremely important to perform safe diagnosis, healthy treatment planning and consistent treatment outcome evaluation in order to understand and cure the complexities of chronic diseases such as Cancer. Although the process of segmenting the desired region accurately is highly challenging and complicated but it has gained enormous importance and several studies have been conducted in improving the accuracy of this task [7][10][12].

Results obtained from the various image segmentation techniques are quite useful in obtaining features of segmented tumor region such as area, volume, perimeter, eccentricity, bounding box and tumor orientation [13]. Numerous research work has been done, and several algorithms are proposed with the goal of detecting the position and boundary of tumors automatically and eventually providing sufficient information to the clinicians, so they can carry out further diagnosis at their earliest. The study presented in this paper reviews the several prominent methods and techniques of automatic segmentation of brain tumor from the MRI images. The rest of the paper and its sections are arranged in the subsequent manner: The 2 nd section presents a generic methodology adopted in the techniques implemented for brain tumor segmentation, followed by a detailed Literature survey in the 3rd section. Section V demonstrates the overall comparison and evaluation of the results followed by the final conclusions and future recommendations in the ending sections.

\section{Methodology}

The general methodology adopted in order to implement the techniques is portrayed in Figure 2. Each step of the methodology is modified according to a specific algorithm into consideration. 


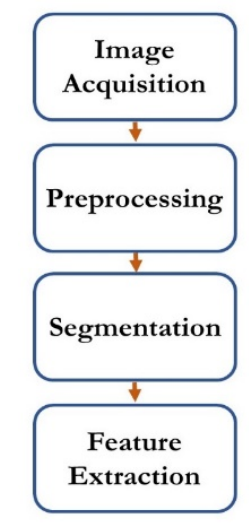

Figure 2: A generalized workflow of image processing.

\section{- Image Acquisition:}

To work with any kind of image processing task, it is essential to first acquire the image to apply the processing on.

\section{- Pre-processing:}

Generating images from various medical imaging techniques may incur unnecessary noise into the image. Hence, any MRI, CT Scan, Mammographic Image or etc., usually comes with a lot of noise. This noise can act as a hurdle when segmenting tumor region from the given input image. To eliminate this issue, an image is first preprocessed in order to remove unwanted outliers, and then sent for further processing. This preprocessing step may include techniques like noise removal, filter application, image enhancement, normalization, etc.

\section{- Segmentation:}

This is the most crucial and core step in applications like tumor detection. It is a simple phenomenon of dividing an image into different meaningful segments to further interpret them. Segmentation of an image can be achieved in numerous ways as already discussed in the literature.

\section{- Feature Extraction}

Once the image has been divided into segments, a post processing step is needed in order to sharpen any edges and blur any unwanted details. This step is called feature extraction where some of the features from the image are extracted for analysis. This will enhance the tumor region so that the area calculation or further operations can be applied on it for more effective results. Morphological operations, edge detection techniques or histogram equalization are most widely used feature extraction steps.

\section{Segmentation Techniques}

MR Images contain a high amount of data. This makes the task of interpreting those scans laborious and tedious even for a radiologist and clinical imaging specialist. In addition, the interpretation results could be different depending upon the experience of the particular specialist [14]. Moreover, various imaging systems may introduce noise in the images, hence making it difficult to segment brain tumor and give an acceptable performance. Segmentation is important as it can help in calculation of quantitative measure of tumor affected part in the brain which is essential for treatment of patient and follow up of the disease. 
The ultimate goal and foremost aim of large number of computer vision, image processing and machine learning based applications is to identify and extract the important patterns or vital features from the image data; using which as a model or reference an imperative description, valuable interpretation or fundamental understanding of the incident, situation or scene can be narrated by the machine for further detailed explanation [8][14][15]. Real time diagnosis of brain tumors and complex diseases from radiographs (as obtained through $\mathrm{X}$-ray, $\mathrm{CT}$ scan, $\mathrm{PET}, \mathrm{MRI}$ and bone-scan etc) is one of the most important but highly challenging task due to high-time consumption and distortion among these images. In order to assist clinicians in decision making, the main focus of the various research groups is to present reliable algorithms which can perform extraordinarily towards accurate segmentation and thus lead to construct a robust as well as to ensure a safe diagnosis system. Table 1 shows a few, most widely used techniques developed by the digital image processing community for image segmentation.

\begin{tabular}{|c|c|}
\hline Segmentation Types & Techniques \\
\hline Region Based & Histogram Threshold, Watershed etc. \\
\hline $\begin{array}{c}\text { Supervised Learning } \\
\text { (classifiers) }\end{array}$ & $\begin{array}{c}\text { Convolutional Neural Network, Support Vector } \\
\text { Machines etc. }\end{array}$ \\
\hline Unsupervised Learning & Fuzzy C-menas, K-means, etc. \\
\hline
\end{tabular}

\section{- Region Based Technique}

Region-based segmentation is a way of determining and locating the desired region correctly. Simply, it combines the individual pixels in an input image to sets of pixels called regions that might correspond to an object or a meaningful part of one. Tanuja and Subhangi [16] came up with a system to segment tumors from the given MRI based on the similarity among the pixels. This idea was called region growing segmentation. It is named so as the basic idea was to define a seed pixel and move to neighboring pixels, grouping the pixels with the similar attributes. The experiments and analysis depict that this method was fast and accurate.

The two most commonly used region-based techniques include Histogram Thresholding and Watershed segmentation. The techniques are discussed in the following section.

\section{- Histogram Thresholding}

Histogram thresholding is widely used region-based segmentation approach that can be used to segment brain tumors among MRI images. In this approach, a threshold is established that could be used to segment the interior area or surface from other organs in the radiographic image dataset [16]-[19]. In principle, in thresholding approach every single pixel is compared to that particular established threshold. If the pixel depicts a value higher than its corresponding threshold, then the pixel is assigned a white color and called "foreground", conversely if the pixel retrieves a value lower or equal to its corresponding threshold, a black color is assigned and titled as "background". Most of the prevailing thresholding approaches are based on bi-level practices, in which an image is categorized into object segments and background. However, MR images, due to their high resolution and distortion issues make these methods non-applicable and difficult to perform smoothly. Consequently, it leads to loss of important information and crucial features from the images which compromise and hampers the overall diagnosis system and 
Muhammad Zawish, Asad Ali Siyal, Shahzad Hyder Shahani, Aisha Zahid Junejo, Aiman Khalil; Brain Tumor Segmentation through Regionbased, Supervised and Unsupervised Learning Methods: A Literature Survey. Journal of Biomedical Engineering and Medical Imaging, Volume 6, No 2, April (2019), pp 8-26

may mislead the medical physicists and radiologists to perform related clinical tasks and decision making [20].

On the other hand, in the watershed approach following three major steps are followed: (i) Initially, the brain is classified into two major halves around its central axis, and finally the histogram is drawn for each of corresponding part, which helps to detect the contagious lesions of the brain. (i) Secondly, the two histograms are compared and the corresponding threshold point values of their histograms are calculated for further evaluation. (iii) Finally, the physical dimensions and morphological features of tumors are evaluated by cropping the detected output image along its corresponding contours.

\section{- Watershed Segmentation}

In region-based segmentation, the mathematical morphological watershed is another most commonly used segmentation approach which segments the organs and tumor lesions based on the watershed ridge lines present in an image. In MR images or even other form of radiographs related to brain tumors, the brain tumors depict quite higher intensity and density and sometimes distortion effects, which makes it extremely difficult to segment the lesions precisely. In such cases, the watershed segmentation is one of the best and potential approach to not only segment and classify the tumors and high density/intensity tissues but also to overcome the distortion problems. Besides, the watershed approach also suffers from over-segmentation and under segmentation constraints because of distortion, noise and various other abnormalities in the radiographs or medical images. To overcome the over-segmentation limitations, the researchers have introduced a method based on the concept of controlled markers in literature. The proposed method deals with the watershed ridge lines and the catchment basins present in an image and consider them a surface wherever the light pixels are low. At first the medical images are converted into gray-scale images and some pre-processing is performed to remove noise followed by marker selection and segmentation etc. In principle, a gradient magnitude is calculated and finally the internal and external markers are calculated to distinguish the foreground of adjacent objects and extract the information from the medical images.

Watershed procedure is widely used for segmentation due to its implementation ease and space and time efficiency. It was first proposed by Digabe and Lantuejou[25], and improved by Beucher and Lantucjou [26]. The major drawback that occurs with this type of segmentation is a phenomenon called over segmentation [27]. In order to overcome this limitation, a marker-based approach was used in [28][29][30] where a brain atlas was used to detect the internal and external markers for division of foreground and background pixels. Different combination of features including color, texture, edge, orientation etc., were tested. All of them except for the combination of color and orientation, gave acceptable and good results.

Swe Zin Oo [23] used the concept of skull stripping, that is, removing any non-brain tissues from the brain image. He coupled this preprocessing technique with the conventional watershed segmentation approach. The study was not only aimed at detecting the tumor region, but also assisted in calculating the volume of the tumor resident in the brain. This volume could be essential in figuring out the grade of the tumor.

Recently, Subudhi et. al., developed an automated watershed-based lesion segmentation (WLSA) method for efficiently delineating the infarct lesions in MRI images of the brain strokes. The method incorporated 
watershed transformation with guided filter through relative fuzzy connectedness (RFC) to distinguish the lesion boundaries reliably and appropriately. The suggested method achieved better results in delineating the lesions and improved the accuracy of detection of ischemic stroke lesions. The proposed technique could be extensively used for early, precise and accurate delineation of stroke lesions in clinical settings [70][71].

\section{- Segmentation with Classifiers (Supervised Learning)}

With the on-going research focus on automating the time-consuming segmentation methods, the classifiers are giving very encouraging results. The classifier technique basically works in two-way fashion. In first stage, it separates amongst typical and anomalous while in next stage, it organizes the kind of variation from the norm in benevolent or dangerous tumor [31]. Below discussed are the two widely used classifiers.

\section{- Convolutional Neural Network (CNN)}

With the ongoing advancement in computational sciences and plethora of application in machine learning, the Convolutional neural networks (CNNs) have gained enormous recognition for variety of image processing applications including automatic medical image segmentation particularly. As image segmentation is a scheme to separate an image (containing valuable information) into various different parts (such as large sets of pixels, or commonly known as super-pixels). The foremost and fundamental objective of segmentation as well as computational image processing is to design and introduce as simpler algorithms as possible which can detect and process the images and analyze the information stored in them (such as features, objects, patterns, lesions and tumors etc.) in more robust, meaningful, understandable and convenient way. Using CNN architectures as a model such valuable information from medical images and radiographs can be readily extracted out with greater accuracy and improved performance with time.

CNN is a discriminative model which directly learns from annotated images without any prior knowledge [32]. CNN based frameworks involve the use of training dataset to instruct a network; and accordingly using these trained networks not only we can predict the class labels but also extract out the important features (such as patterns, edges, lines) and further train the other set of classifiers. Using this strategy, the patches of the information (as an input) is extracted out from the MRI images which are processed through convolution based filters and local sub-sampling to obtain the highly complex features (such as patterns, edges, lines) and help to yield the location and size of the tumors based on their corresponding computed class scores. Moreover, the CNN architectures have also advantage of automatic learning the complex features related to healthy tissues as well abnormal tissues (tumors) directly acquired from MRI images.

In order to contribute to the domain, Lang, Zhao and Jiya [33] proposed an architecture of convolutional neural networks to solve manual segmentation issues. Low resolution and noisy images such as CT scan and MRI are dealt easily by powerful classification features of CNN. The study presented three different CNN architectures of $5 \times 5,12 \times 12$ and $28 \times 28$ patch sizes. As a result of comparison of the three architectures proposed, it was found out that the architecture with $28 * 28$ input patch size was the most accurate one. 
Muhammad Zawish, Asad Ali Siyal, Shahzad Hyder Shahani, Aisha Zahid Junejo, Aiman Khalil; Brain Tumor Segmentation through Regionbased, Supervised and Unsupervised Learning Methods: A Literature Survey. Journal of Biomedical Engineering and Medical Imaging, Volume 6, No 2, April (2019), pp 8-26

Similar architecture was adopted by Mengqiao, Yilie and Hao. They built a 22-layer deep learning architecture based on convolutional neural networks [34]. The architecture consisted of cascaded convolutional layers of size $3^{*} 3^{*} 2$ instead of layers of size $7 * 7 * 4$ as the effect of both is the same and the former has lesser weights. Besides convolutional layers, the architecture consisted of fully connected layers and the average pooling layers. The results were evaluated on BRATs 2015 dataset with the help of an online platform.

Recently, various machine learning as well as deep learning based approaches have been widely implemented in brain tumor segmentation based experimental and research studies after their highly dominative applications and commendable progressive success in numerous other image analysis domains, such as semantic segmentation [53] - [55], images classification [56] and objects detection [57]. Among these deep learning techniques, the newly developed CNNs based tumor segmentation method such as 2D-CNNs [58] - [61] and 3D-CNNs [62] - [64] achieved better results and overall performance compared to other reported techniques.

Most recently, Zhao et. al., developed a new deep learning based brain tumor segmentation system by involving the dynamic fusion of conditional random fields (CRFs) and fully convolutional neural networks (FCNNs) in an automated and robust unified framework. Their newly proposed method is capable of segmenting the brain images slice-by-slice, yielding better, spatially consistent and quite faster results than compared to other contemporary techniques such as image patch based segmentation [65].

\section{- Support Vector Machines (SVM}

SVM has been considered as one of the consistent and best methods for classifying the features, patterns or objects present inside the images. Using SVM, the set of images are basically divided into two / various resultant classes. The classification is usually performed by finding the hyper-plane principle that freely differentiates the two classes perfectly as shown in Figure 3 below.

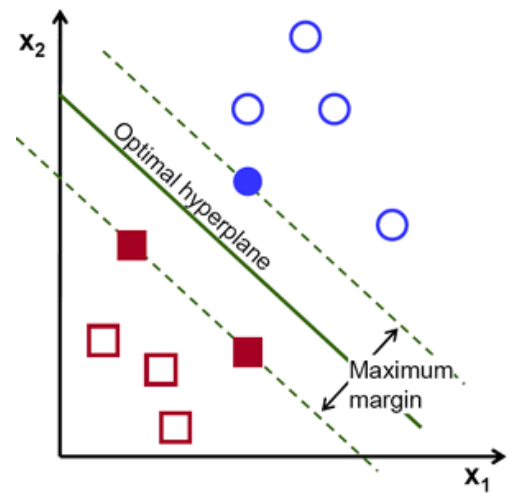

Figure 3: Hyperplane Classification Principle.

It constructs a hyper plane adopting a kernel function [13][35]. As presented in Figue.2, the feature vectors indicated on the left side of the main hyper plane belong to the class -1 , whereas the feature vectors designated on the right side of the main hyper plane corresponds to the class +1 .

The segmentation with SVM mainly depends upon the following phases (a) feature extraction from training image (b) the selection of SVM model (c) preparation of data-set (d) SVM training [34][36]. After performing the pre-processing and the training steps, the patterns (such as intensity and position) are 
extracted out automatically from the test image which is consigned as an input to the SVM model. On the other hand, in the segmentation phase, the test feature vector is compared with the trained feature vector and based on the comparison the abnormal brain tissues (tumor) are segmented out from the consigned input image.

A. Kumar, B. Mahavir and Richika in their study [43] used Support vector machine, coupled with K-means clustering and Principle Component Analysis for extraction and classification of the tumor region inside the brain. In the methodology given the data including brain scans were trained using support vector machine while the tumor was segmented using k-means and PCA. The purpose of training the data through SVM classifier was to find out the class of the tumor detected. This approach had an accuracy of 96\\% for tumor volume detection as depicted by the results. Besides segmenting the tumor region, the paper also gave a detailed information on K-means and PCA, along with the relation between them.

Similarly, G. Gupta and V. Singh also worked on SVM for classification of images, coupled with Fuzzy CMeans. Their approach was to first skull strip the input image, apply FCM to segment the image and finally use SVM to further classify the images which gave more enhanced and better results [44].

\section{- Segmentation with Unspervised Learning}

Unlike supervised learning, in these algorithms we are not required to provide the prior knowledge, the algorithms are left on their own to find the insights from the given image.

More precisely, the image segmentation with unsupervised learning is a process in which an image is automatically divided into various different homogeneous sections based on their corresponding similarity measures. It is easier to segment using unsupervised learning methods as supervised increase the computational cost by demanding the machine to be trained first and then tested [52]. Clustering is one of the vital implementations of unsupervised learning, our study provides the review of 2 algorithms based on clustering i.e. Fuzzy C-Means and K-Means.

\section{- Fuzzy C-Means}

Clustering approach has been widely used in different computational domains such as machine learning, computer vision and image processing.

Besides, clustering technique has also recently progressed into various biomedical and healthcare applications predominantly for the detection of abnormal brain tissues (tumors) from radiographs acquired through magnetic resonance imaging (MRI) modality [37]. Dunn et al., introduced a clustering based Fuzzy c-means (FCM) approach, that was further enhanced by Bezdek et a., which greatly furthered the segmentation proficiency and was proved to be a better method compared previous reported techniques in the literature. Clustering with FCM is done in a way that it bifurcates one group of data into two or various different corresponding clusters, as presented in Figure 4. 
Muhammad Zawish, Asad Ali Siyal, Shahzad Hyder Shahani, Aisha Zahid Junejo, Aiman Khalil; Brain Tumor Segmentation through Regionbased, Supervised and Unsupervised Learning Methods: A Literature Survey. Journal of Biomedical Engineering and Medical Imaging, Volume 6, No 2, April (2019), pp 8-26

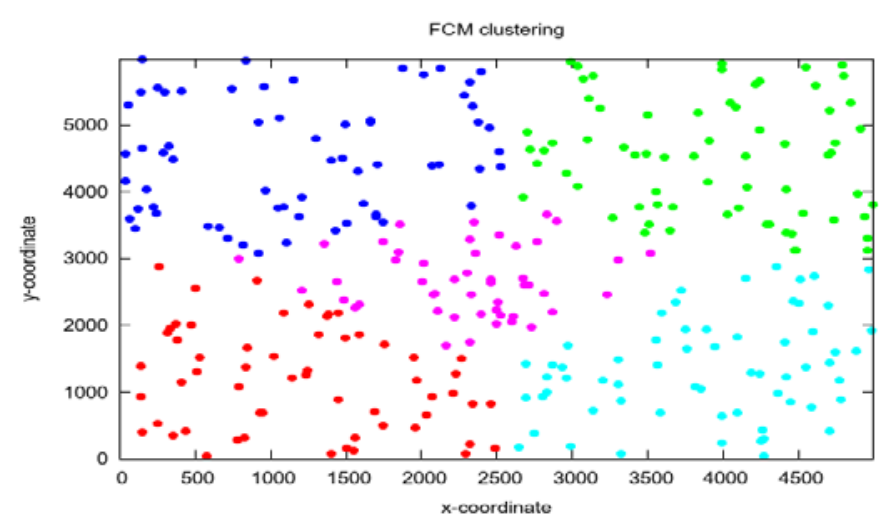

Figure 4: Clustering with FVM.

This method is basically executed for pattern recognition based applications. In this method, the membership value is allocated to each of the related data point that corresponds to each cluster center based on the distance between each of the cluster and data point. The possibility of the membership value towards any particular cluster is totally based on the closeness of the data to cluster center. The analysis of brain tumor segmentation suggests that by means of an unsupervised FCM clustering algorithm, the segmentation of brain tumor will be resulted in active cells, necrotic core and edema [4]. With respect to computational rate, the FCM algorithm will be more effective when the cluster center and membership value updating criterion is altered.

In 2016, Suganya and Shanthi reviewed Fuzzy C Means algorithm and its various applications in medical imaging, pattern recognition, bioinformatics and data mining [38]. The basic objective of the study was to analyze different algorithms based of FCM clustering and bring into light the merits and demerits of each.

Recently, Pham et. al., developed a novel clustering algorithm by integrating fuzzy entropy clustering for segmentation of the abnormal brain tissues (tumors) from MRI images. The newly suggested approach is basically based on the improved particle swarm optimization (PSO) algorithm, LHNPSO algorithm and kernelized fuzzy entropy clustering with modified fitness function to efficiently segment the brain tumors from MRI images by partially overcoming two serious issues, the first is sensitivity to noise and the other is INU artifact [66].

Kumar et. al., suggested a modified intuitionistic fuzzy c-means algorithm (MIFCM) to analytically solve the optimization problem and objective function using Lagrange method of undetermined multipliers. The proposed MIFCM method has allowed to segment brain MRI data by overcoming the limitations of noise and imprecise measurement [67].

Shanmuga Priya et. al., anticipated FCM based multilevel segmentation by combining fuzzy c-means, skull tripping and graph cut methods for detecting the tumor tissues and edema among brain MRI images. The clustering process has been enhanced by merging multiple kernels based on the spatial information to perform efficient segmentation [68].

\section{- K-Means}

The recognized clustering problem can be solved by K-means which is the simplest in unsupervised learning algorithms. There are four steps in a standard K-means algorithm, these are: initialization, 
classification, computational and convergence condition. The process initializes by dividing a particular data set into stable positive $\mathrm{K}$ number of clusters so that $\mathrm{k}$ centroids can be defined as one for each cluster. As the results rely on the locations, therefore these centroids should be located in an elusive technique. One of the best ways, is to place them at a far distance from each other. The next step follows by selecting a point which is in acquaintance to a given data set and change it to the closest centroid. The first group age is complete when first step is finished which only happens when no any point exists the last. The outcome of first step results in $\mathrm{K}$ new centroids of the clusters which should be recalculated at the end of the first step. Once new $\mathrm{K}$ centroids are received, there is a need of a new connection between the same data set points and the nearest new centroid. Therefore, a loop is created which helps in analyzing the changed location of $\mathrm{K}$ centroids in each phase unless all centroids come to static. In other words, centroids do not move any more shown in Figure 5.

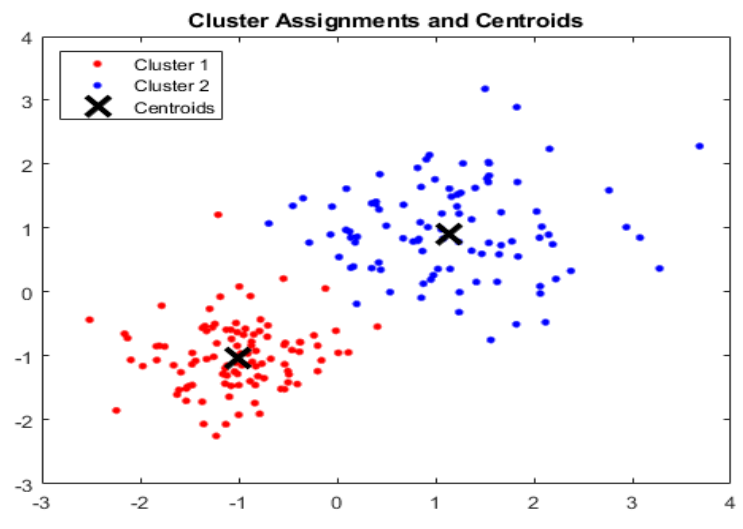

Figure 5: Clustering though K-means.

In [52] Vijay and Subhashini took into consideration the problem of labeling in image segmentation specially when it comes to automated brain tumor detection. They proposed a methodology that used morphological operations for preprocessing and traditional K-means technique with a slight modification that was to reduce the number of iterations required for proper clustering by suggesting the computed distance between a cluster center and data point under examination, which is stored in a data structure. This combination of K-means clustering, and morphological operations produced $95 \backslash \%$ accurate results on a sample space of 100 input images, as depicted in the results.

Dr. Patil, Dr. Jain and Pachpande proposed a computer aided application to segment tumor from the given MRI scans [25]. The segmentation idea adopted for the study worked with an amalgamation of K-means clustering and Fuzzy $\mathrm{C}$ means based clustering approaches. Four different modalities of images were tested for experiments and the results were generated based on parameters like, Mean Square Error (MSE), Contrast, Correlation, Max error, Area, etc. The study and results concluded that the method proposed was robust, accurate and time saving.

\section{Comparative Studies}

Where some researchers have been making remarkable contributions to the domain, there, on the other hand, other researchers are comparing different techniques produced and tested. In this regard, some worthy contributions are made in [40][45][51] and others. 
Muhammad Zawish, Asad Ali Siyal, Shahzad Hyder Shahani, Aisha Zahid Junejo, Aiman Khalil; Brain Tumor Segmentation through Regionbased, Supervised and Unsupervised Learning Methods: A Literature Survey. Journal of Biomedical Engineering and Medical Imaging, Volume 6, No 2, April (2019), pp 8-26

Said and Ibrahim [40] presented a study to compare various segmentation algorithms. A MATLAB toolbox was proposed in the paper to compare K-Means [25], Fuzzy C Means [38], Region-growing [41] and Otsu [42] techniques. Said and Ibrahim used Artificial Neural Networks (ANN) for classification and accuracy of the techniques compared. According to the results, K-Means and Fuzzy C Means algorithms had the same accuracy i.e. $96.7 \backslash \%$, which was higher than the rest of the algorithms. Otsu had second highest accuracy of upto $90 \backslash \%$ whereas region growing method was least accurate.

Another comparative study produced by Suhasini and Vijaykumar [45] compared the studies like Support Vector Machine Classifier, Fuzzy C Means [46], K-means [47] Hybrid Clustering [48], Mathematical Morphology [49] and Integrated Bayesian Model [50] and more. These various techniques were experimentally compared based on the accuracy. Morphological filtering was regarded as the highest accuracy algorithm with an accuracy of $99 \backslash \%$ followed by Hybrid clustering, FCM and Bayesian. K-means clustering was also one of the highlights of the study as it had the greatest number of advantages in terms of implementation and usage easiness. Though morphological filtering had the most accuracy, but it required a high computational power as compared to the K-means which had minimum implementation hassle and second highest accuracy.

G. Rao and B. Srinivas in [51] contributed to the domain by contrasting Fuzzy C-Means and K-Means clustering techniques. Segmentation through FCM and K-Means was compared with respect to its Mean Square Error (MSE), Peak Signal to Noise Ratio (PSNR), Peak Time (PTime) and area calculation. Results of the study showed that FCM had a greater accuracy of approximately $93 \backslash \%$, along with lower PTime in comparison to K-Means, which had about 76\\% accuracy only. accurate.

\section{Discussion}

Image Segmentation is one of the most fundamental concepts in computer vision. Segmentation aims to change/modify the representation of an image to extract meaningful information from it. This paper presents a comprehensive review of literature on various segmentation methods that are currently being worked upon for brain tumor segmentation. A number of algorithms and hybrid approaches for all these methods have been presented in Section III. Besides this, various automated and semi-automated techniques have also been discussed which on real time implementation will be able to contribute the excellence of computer technology to assist in the field of medical science. Figure 6 shows the results of a few algorithms discussed. 


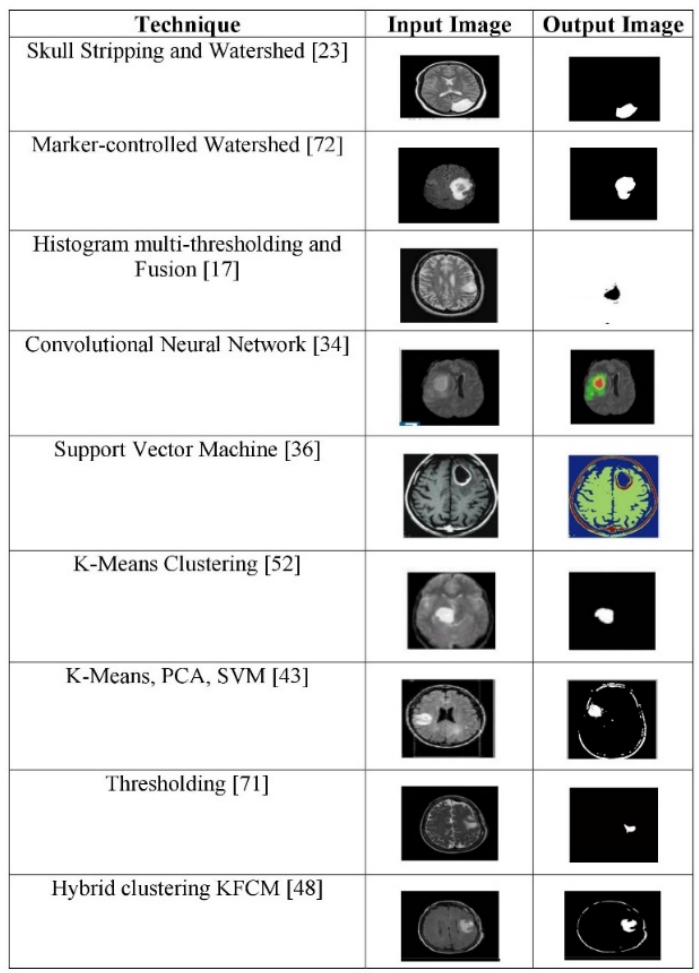

Figure 6: An overview of different brain tumor segmentation techniques.

Despite of the huge contributions being made; none of systems has perfected to be accepted in real time clinical applications.

The study presented in the paper suggests that the supervised learning methods have better accuracy, but they are heavy in terms of computational power i.e. consume more computational cost, storage space and processing time while gradient based methods as well as unsupervised methods are accurate and require lesser resources. In other words, some methods have an easier implementation while others have a greater accuracy. One thing is achieved at the cost of another hence this domain still has a lot of area for further improvement. The researchers should aim at creating systems utilizing minimum use of resources but producing better results.

Though computer aided systems are difficult to implement and update, and it requires computer literates to operate such systems, but they are not as laborious as manual methods.

\section{Conclusion and Future Perspectives}

In this paper, a number of brain tumor segmentation algorithms are presented. It can be concluded from the study that these automated systems lack in factors like interoperability and easy handling of tools and hence are not yet clinically accepted. Also, for real life acceptance, these systems need to have more accuracy and must be able to calculate the volume of tumor to know the stage of the tumor. Moreover, they must also be able to classify multiclass tumors and estimate tumor progression.

This kind of developments in supervised learning architectures and/or the hybrids of supervised and unsupervised may aid in standardizing the current methods which will in turn help in clinical acceptance 
Muhammad Zawish, Asad Ali Siyal, Shahzad Hyder Shahani, Aisha Zahid Junejo, Aiman Khalil; Brain Tumor Segmentation through Region-

based, Supervised and Unsupervised Learning Methods: A Literature Survey. Journal of Biomedical Engineering and Medical Imaging, Volume 6, No 2, April (2019), pp 8-26

of these automated systems, resulting in another major contribution of computer technology in medical science.

\section{CONFLICT OF INTEREST}

The authors declare no conflict of interest.

\section{AUTHORS CONTRIBUTION}

All authors contributed equally to this work. All authors wrote, reviewed, and commented on the manuscript. All authors have read and approved the final version of the manuscript.

\section{ACKNOWLEDGEMENTS}

The authors of this paper would like to acknowledge and thank Mehran University of Engineering and Technology to have supported the study by providing all the necessary resources.

\section{LIST OF ABBREVIATIONS}

$\begin{array}{lll}\text { MRI } & = & \text { Magnetic resonance imaging } \\ \text { CT } & = & \text { Computed tomography } \\ \text { GM } & = & \text { White matter } \\ \text { WM } & = & \text { Cerebrospinal fluid } \\ \text { CSF } & = & \text { Watershed based lesion segmentation } \\ \text { WLSA } & = & \text { Relative fuzzy connectedness } \\ \text { RFC } & = & \text { Convolutional neural network } \\ \text { CNN } & = & \text { Fully convolutional neural network } \\ \text { FCNN } & = & \text { Artificial neural network } \\ \text { ANN } & = & \text { Conditional random fields } \\ \text { CRF } & = & \text { Support vector machine } \\ \text { SVM } & = & \text { Principal component analysis } \\ \text { PCA } & = & \text { Fuzzy c-means } \\ \text { FCM } & =\quad \text { Particle swarm optimization } \\ \text { PCO } & =\quad \text { Modified intuitionistic fuzzy c-means algorithm } \\ \text { MIFCM } & =\quad \text { Mean square error } \\ \text { MSE } & =\quad \text { Signal to noise ratio } \\ \text { SNR } & \text { Peak signal to noise ratio } \\ \text { PSNR } & & \end{array}$




\section{REFERENCES}

[1] Richa Aggarwal, Amanpreet Kaur. (2012) “Comparative Analysis of Different Algorithms For Brain Tumor Detection", International Journal of Science and Research (IJSR).

[2] Oelze, M.L,Zachary, J.F. , O'Brien, W.D., Jr., -Differentiation of tumor types in vivo by scatterer property estimates and parametric images using ultrasound backscatter — , on page(s) :1014 - 1017 Vol.1, 5-8 Oct. 2003 .

[3] Pooja Thakur, et al. (2015). Brain Tumor Detection, Segmentation using watershed segmentation and Morphological operations. International Journal of Advanced Research in Electronics and Communication Engineering (IJARECE).

[4] Gursangeet Kaur and Jyoti Rani (June 2016). MRI Brain Tumor Segmentation Methods- A Review. International Journal of Current Engineering and Technology.

[5] N.S.Zulpe, S.S.Chowhan."Statical Approach For MRI Brain Tumor Quantification". International Journal of Computer Applications(0975-8887), vol.35-No.7, December 2011.

[6] D. Martin, C. Fowlkes, D. Tal, and J. Malik, -A database of human segmented natural images and its application to evaluating segmentationalgorithms and measuring ecological statistics, $\|$ in Proc. 8th Int. Conf. Computer Vision, Jul. 2001, vol. 2, pp. 416-423.

[7] J. Freixenet, X. Munoz, D. Raba, J. Marti, and X. Cufi, -Yet another survey on image segmentation: Region and boundary information integration, $\|$ in Proc. 7th Eur. Conf. Computer Vision Part III, Copenhagen,Denmark, May 2002, pp. 408-422, LNCS.

[8] H. Tang, E.X. Wu, Q.Y. Ma, D. Gallagher, G.M. Perera, and T. Zhuang, -MRI brain image segmentation by multi-resolution edge detection and region selection, $\|$ Computerized Medical Imaging and Graphics, vol. 24, pp. 349-357, 2000.

[9] Mohammad Shajib Khadem, "MRI Brain image segmentation using graph cuts", Master of Science Thesis in Communication Engineering, Department of Signals and Systems, Chalmers University Of Technology, Goteborg, Sweden, 2010.

[10] Smita Pradhan, "Development of Unsupervised Image Segmentation Schemes for Brain MRI using HMRF model", Master Thesis at Department of EE, NIT, Rourkela, 25 Mar 2010, pp. 4-6.

[11] Roopali R.Laddha et al, A Review on Brain Tumor Detection Using Segmentation And Threshold Operations. (IJCSIT) International Journal of Computer Science and Information Technologies, Vol. 5 (1) , 2014, 607-611.

[12] T. Logeswari, M. Karnan, -An improved implementation of brain tumor detection using segmentation based on soft computing\|, Page(s): 006-014, Journal of Cancer Research and Experimental Oncology Vol. 2(1), March 2010.

[13] T. Sathies Kumar., et al. (2017). Brain Tumor Detection Using SVM Classifier. IEEE 3rd International Conference on Sensing, Signal Processing and Security (ICSSS). 
Muhammad Zawish, Asad Ali Siyal, Shahzad Hyder Shahani, Aisha Zahid Junejo, Aiman Khalil; Brain Tumor Segmentation through Regionbased, Supervised and Unsupervised Learning Methods: A Literature Survey. Journal of Biomedical Engineering and Medical Imaging, Volume 6, No 2, April (2019), pp 8-26

[14] Vida Harati, Rasoul Khayati, Abdolreza Farzan, "Fully automated tumor segmentation based on improved fuzzy connectedness algorithm in brain MR images,"Elsevier Itd, 2011.

[15] Gonalves, P. C.; Tavares, J. M. R.; Jorge, R. N.: Segmentation and simulation of objects represented in images using physical principles. Computer Modeling in Engineering - Sciences, vol. 32, no. 2, pp. 4555(2008).

[16] Orlando J. Tobias and Rui Seara," Image Segmentation by Histogram Thresholding Using Fuzzy Sets," IEEE transactions on Image Processing,Vol. 11,NO. 12,PP-1457-1465,DEC 2002.

[17] F.kurugollu, "color image segmentation using histogram multithresholding and fusion," Image and Vision Comuting,Vol. 19,pp915-928,2001.

[18] Jichuan Shi, -Adaptive local threshold with shape information and its application to object segmentation\|, Page(s)1123 - 1128, Robotics and Biomimetics (ROBIO), 2009 IEEE International Conference,1923 Dec. 2009.

[19] Roopali R.Laddha, S.A.Ladhake (2014), A Review on Brain Tumor Detection Using Segmentation And Threshold Operations, International Journal of Computer Science and Information Technologies, Vol. 5 (1), 607-611.

[20] Alyaa H. Ali, Kawther A.Khalaph, Ihssan S.Nema. Segmentation of brain tumour using Enhanced Thresholding Algorithm and Calculatethe area of the tumour. IOSR Journal of Research \& Method in Education (IOSR-JRME) e-ISSN: 2320-7388,p-ISSN: 2320-737X Volume 4, Issue 1 Ver. II (Jan. 2014), PP 58-62.

[21] Saif D. Salman and Ahmed A. Bahrani, "Segmentation of tumor tissue in gray medical images using watershed transformation method," Intl. Journal of Advancements in Computing Technology,Vol. 2, No. 4,pp123-127,OCT 2010.

[22] Gang Li , -Improved watershed segmentation with optimal scale based on ordered dither halftone and mutual information\|, Page(s) 296 - 300, Computer Science and Information Technology (ICCSIT), 2010 3rd IEEE International Conference, 9-11 July 2010.

[23] Swe Zin Oo, Aung Soe Khaing (2014), Brain tumor detection and segmentation using watershed segmentation and morphological operation, International Journal of Research in Engineering and Technology, elSSN: 2319-1163 | pISSN: 2321-7308.

[24] Roshan G. Selkar, Prof. M. N. Thakare, "Brain Tumor Detection And Segmentation By Using Thresholding and Watershed Algorithm", IJAICT, vol 1, 2014.

[25] Patil, A. J., et al. (2005). Pachpande, Automatic Brain Tumor Detection Using K-Means, 13896-13903.

[26] H. Digabel C. Lantuejoul "Iterative algorithms" Quantitative Analysis of Microstructures in Materials Sciences Biology and Medicine pp. 85-99 1977.

[27] S. Beucher C. Lantuejoul "Use of watersheds in contour detection" 1979. 
[28] Amoda, N. \& Kulkarni, R. K. Image segmentation and detection using watershed transform and region based image retrieval. Int. J. Emerg. Trends \& Techno. Comp. Sci. 2, 89-94 (2013).

[29] C. C Benson V. L Lajish Kumar Rajamani "Brain Tumor Extraction from MRI Brain Images Using Marker Based Watershed Algorithm" International Conference on Advances in Computing Communications and Informatics (ICACCI) pp. 318-323 2015.

[30] Rivest, J., Beucher, S., Delhomme. J, -Marker-controlled segmentation: an application to electrical borehole imaging\|, Journal of Electronic Imaging, April pp. 136-142, 1992.

[31] A. Sankari and S. Vigneshwari. "Automatic tumor segmentation using convolutional neural networks". (2017) Third International Conference on Science Technology Engineering \& Management (ICONSTEM).

[32] Wang Mengqiao,. et al. (September 2017). "The multimodal brain tumor image segmentation based on convolutional neural networks". (ICCIA).

[33] R. Lang, L. Zhao and K. Jia, "Brain tumor image segmentation based on convolution neural network," 2016 9th International Congress on Image and Signal Processing, BioMedical Engineering and Informatics (CISPBMEI), Datong, 2016, pp. 1402-1406.

[34] W. Mengqiao, Y. Jie, C. Yilei and W. Hao, "The multimodal brain tumor image segmentation based on convolutional neural networks," 2017 2nd IEEE International Conference on Computational Intelligence and Applications (ICCIA), Beijing, 2017, pp. 336-339.

[35] Nguyen Thanh Thuy, Tran Son Hai, Le Hoang Thai, "Image Classification using Support Vector Machine and Artificial Neural Network", Vietnam.

[36] Swapnil R. Telrandhe,. et al. "Detection of brain tumor from MRI images by using segmentation \& SVM". 2016 World Conference on Futuristic Trends in Research and Innovation for Social Welfare (Startup Conclave).

[37] Benson. C. C., et al. "Brain Tumor Segmentation from MR Brain Images using Improved Fuzzy c-Means Clustering and Watershed Algorithm". 2016 Intl. Conference on Advances in Computing, Communications and Informatics (ICACCI), Sept. 21-24, 2016, Jaipur, India.

[38] Suganya, R., \& Shanthi, R. (2012). Fuzzy C- Means Algorithm - A Review, 2(11), 1-3.

[39] Ali Salem Bin Samma and Rosalina Abdul Salam. "Adaptation of K-Means Algorithm for Image Segmentation". World Academy of Science, Engineering and Technology 502009.

[40] Said, Ashraf \& Sayed, Fatma. (2017). Comparative Study of Segmentation Techniques for Detection of Tumors Based on MRI Brain Images. International Journal of Bioscience, Biochemistry and Bioinformatics. 8. 10.17706/ijbbb.2018.8.1.1.

[41] Tanuja, M., \& Shewale, P. (2016). Detection of Brain Tumor Based On Segmentation Using Region Growing Method, 5(2), 173-176 
Muhammad Zawish, Asad Ali Siyal, Shahzad Hyder Shahani, Aisha Zahid Junejo, Aiman Khalil; Brain Tumor Segmentation through Regionbased, Supervised and Unsupervised Learning Methods: A Literature Survey. Journal of Biomedical Engineering and Medical Imaging, Volume 6, No 2, April (2019), pp 8-26

[42] Otsu, N. (1979). A threshold selection method from gray-level histograms. IEEE Transactions on Systems, Man, and Cybernetics, 9(1).

[43] Kumar, Arun. "A Novel Approach for Brain Tumor Detection Using Support Vector Machine, K-Means and PCA Algorithm." (2017).

[44] Gaurav Gupta, Vinay Singh, "Brain Tumor segmentation and classification using Fcm and support vector machine". International Research Journal of Engineering and Technology (IRJET) e-ISSN: 2395 -0056 Volume: 04 Issue: 05, May-2017.

[45] K Uma Suhasini, V. (December 2016). A Comparative Analysis of Brain Tumor Segmentation Techniques. Indian Journal of Science and Technology, Vol 9(48).

[46] V. Janani, P. M. (May 2013). Image Segmentation For Tumor Detection Using Fuzzy Inference System. International Journal of Computer Science and Mobile Computing, 244- 248.

[47] Sundararaj GK, B. V. (2015). Robust classification of primary brain tumor in computer tomography images using K-NN and Linear SVM. . International Conference on Contemporary Computing and Informatics (IC3I).

[48] Abdel- Maksoud E, E. M.-A. (March, 2015). Brain tumor segmentation using hybrid based clustering techniques. . Egyptian Informatics Journal., 71-81.

[49] Ananda RS, T. T. (May, 2013). Automatic segmentation framework for primary tumors from brain MRIs using morphological filtering techniques. 5th International Conference on Biomedical Engineering and Informatics (BMEI).

[50] Corso JJ, S. E.-S. (May, 2008). Efficient multilevel brain tumor segmentation with Integrated Bayesian model classification. IEEE Transactions on Medical Imaging., 629-40.

[51] G. Rao, B. S. (2018). "Unsupervised learning algorithms for MRI Brain Tumor Segmentation". SPACES. Vijayawada, India: IEEE.

[52] J. Vijay and J. Subhashini, "An Efficient Brain Tumor Detection Methodology Using K-Means Clustering Algorithm", International conference on Communication and Signal Processing, April 3-5, 2013, India.

[53] Liu, Ziwei, et al. "Semantic image segmentation via deep parsing network." Proceedings of the IEEE International Conference on Computer Vision. 2015.

[54] Zheng, Shuai, et al. "Conditional random fields as recurrent neural networks." Proceedings of the IEEE international conference on computer vision. 2015.

[55] Long, Jonathan, Evan Shelhamer, and Trevor Darrell. "Fully convolutional networks for semantic segmentation." Proceedings of the IEEE conference on computer vision and pattern recognition. 2015.

[56] Krizhevsky, Alex, Ilya Sutskever, and Geoffrey E. Hinton. "Imagenet classification with deep convolutional neural networks." Advances in neural information processing systems. 2012. 
Journal of Biomedical Engineering and Medical Imaging, Volume 6, No 2, April 2019

[57] Girshick, Ross, et al. "Rich feature hierarchies for accurate object detection and semantic segmentation." Proceedings of the IEEE conference on computer vision and pattern recognition. 2014.

[58] Pereira, Sérgio, et al. "Brain tumor segmentation using convolutional neural networks in MRI images." IEEE transactions on medical imaging 35.5 (2016): 1240-1251.

[59] Havaei, Mohammad, et al. "Brain tumor segmentation with deep neural networks." Medical image analysis 35 (2017): 18-31.

[60] Dvorak, Pavel, and Bjoern Menze. "Structured prediction with convolutional neural networks for multimodal brain tumor segmentation." Proceeding of the Multimodal Brain Tumor Image Segmentation Challenge (2015): 13-24.

[61] Zikic, Darko, et al. "Segmentation of brain tumor tissues with convolutional neural networks." Proceedings MICCAI-BRATS(2014): 36-39.

[62] Kamnitsas, Konstantinos, et al. "Efficient multi-scale 3D CNN with fully connected CRF for accurate brain lesion segmentation." Medical image analysis 36 (2017): 61-78.

[63] Yi, Darvin, et al. "3-D convolutional neural networks for glioblastoma segmentation." arXiv preprint arXiv:1611.04534(2016).

[64] Urban, Gregor, et al. "Multi-modal brain tumor segmentation using deep convolutional neural networks." MICCAI BraTS (Brain Tumor Segmentation) Challenge. Proceedings, winning contribution (2014): 31-35.

[65] Zhao, Xiaomei, et al. "A deep learning model integrating FCNNs and CRFs for brain tumor segmentation." Medical image analysis 43 (2018): 98-111.

[66] Pham, Thuy Xuan, Patrick Siarry, and Hamouche Oulhadj. "Integrating fuzzy entropy clustering with an improved PSO for MRI brain image segmentation." Applied Soft Computing 65 (2018): 230-242.

[67] Kumar, Dhirendra, et al. "A modified intuitionistic fuzzy c-means clustering approach to segment human brain MRI image." Multimedia Tools and Applications (2018): 1-25.

[68] ShanmugaPriya, S., and A. Valarmathi. "Efficient fuzzy c-means based multilevel image segmentation for brain tumor detection in MR images." Design Automation for Embedded Systems (2018): 1-13.

[69] Subudhi, Asit, Subhranshu Jena, and Sukanta Sabut. "Delineation of the ischemic stroke lesion based on watershed and relative fuzzy connectedness in brain MRI." Medical \& biological engineering \& computing 56.5 (2018): 795-807.

[70] Subudhi, A., Jena, S. \& Sabut, S., "Delineation of the ischemic stroke lesion based on watershed and relative fuzzy connectedness in brain MRI”, Med Biol Eng Comput (2018) 56: 795.

[71] A. Kapil, S. Shukla, "Brain Tumour Extration From MR Images Using Segmentation Techniques: A Review".

[72] A. Junejo, S. Memon, I. Memon, S. Talpur, "Brain Tumor Segmentation Using 3D Magnetic Resonance Imaging Scans", IEEE Internation Conference on Advanced Research in Engineering Sciences, Dubai 2018. 